\title{
RESEARCH HIGHLIGHT Heart muscle regeneration: the wonder of a Cardio-Cocktail
}

\author{
Matthew C Hill ${ }^{1}$ and James F Martin ${ }^{1,2,3}$ \\ Cell Research (2018) 28:503-504; https://doi.org/10.1038/s41422-018-0035-5
}

\begin{abstract}
Mohamed et al. recently reported in Cell a combination of cell cycle-associated genes that can be virally delivered to adult mammalian cardiac tissue in vivo to drive cardiomyocyte proliferation and regeneration in the context of myocardial infarction. With advances in gene therapy, this study uncovers a translational gene combination with therapeutic potential for treating heart failure.
\end{abstract}

The heart, the first organ to develop, begins pumping blood to the embryo at approximately embryonic day 8 (E8) in the mouse. Throughout cardiac morphogenesis, cardiac muscle cells or cardiomyocytes (CMs), perform a plethora of cellular activities, including proliferation, contraction, cell migration and differentiation, leading to the achievement of proper cardiac ontogeny. However, soon after birth, mammalian CMs exit the cell cycle, lose their proliferative capacity, and differentiate. Differentiated CMs possess a contractile apparatus, known as the sarcomere, and are often mutli-nucleated or contain single polyploid nuclei potentially adding further structural hurdles to cell division. CMs grow via hypertrophy as opposed to hyperplasia. Thus, an increase in cell size is responsible for most postnatal cardiac growth. Adult CMs have little to no expression of mitotic genes but they do express inhibitors that prevent cell cycle induction and cytokinesis. Prominent among these potent mitotic inhibitory pathways is the Hippo pathway. ${ }^{1}$ At the epigenetic level, adult CMs also have decreases in chromatin accessibility near cell cycle genes and exhibit hypermethylation of genomic regulatory elements associated with critical developmental genes that initiate proliferation. $^{2}$ Thus, during homeostasis, adult mammalian CMs rarely enter cell cycle.

Following myocardial infarction, CMs are progressively lost, placing a large burden on residual CMs to maintain physiologic cardiac function. Lost CMs are replaced by scar tissue, critical to prevent cardiac rupture, that impedes proper cardiac function and is considered a hallmark of heart failure. One goal of cardiac regenerative medicine is to improve cardiac function through replenishing muscle tissue and resolving scar tissue. Strategies for achieving heart regeneration fall into three main categories: 1) providing exogenous pools of stem cells, 2) reprogramming cardiac fibroblasts into CMs, and 3) promoting expansion of endogenous CMs, the method addressed by Mohammed et al.

Since adult CMs do not express proliferation genes, Mohammed et al. provided these genes exogenously with the thought that this would initiate cell division. To define the minimal number of cell cycle genes required to initiate cell cycle in adult CMs, Mohamed et al. performed transcriptomic profiling of fetal, neonatal, and adult heart tissue. They identified 15 candidate genes enriched in proliferative fetal and neonatal stages, and then overexpressed these genes individually in primary murine CMs using adenoviral vectors and assessed cell proliferation using histone $\mathrm{H} 3$ phosphorylation (PHH3), a marker for mitosis. Among them, cyclindependent kinase 1 (CDK1), cyclin B1 (CCNB), and aurora kinase B (AURKB) top hits for inducing CM proliferation. However, when these three genes were co-expressed in primary murine CMs or human induced pluripotent stem cell-derived CMs (hiPS-CMs), they initiated improper cell cycle re-entry and DNA damage response resulting in cell death. The authors next sought to rationally identify cell cycle genes that could promote proper cell cycle re-entry without DNA damage response and cell death. Via the deployment of a systematic screen, they determined that the combination of CDK1, CCNB, CDK4 and CCND (4 F) could promote $\mathrm{CM}$ proliferation without reducing cell viability.

Immunofluorescence markers to label mitotic cells (e.g., PHH3) cannot track cytokinesis. Mohamed et al. used a tool originally developed by Drosophila geneticists and modified for use in the mouse known as Mosaic Analysis with Double Markers (MADM). ${ }^{3}$ With MADM lineage tracing system, they labeled cells that went through mitosis, as MADM relies on G2 inter-chromosomal recombination. MADM mice possess two chimeric genes each containing $\mathrm{N}$ - and $\mathrm{C}$-terminal portions of red fluorescent protein (RFP) and green fluorescent protein (GFP). Each cell starts out with a copy of non-functional GFP-N:RFP-C and RFP-N:GFP-C, and during $\mathrm{G} 2$, Cre-mediated recombination between homologous chromosomes can generate clones that are GFP single positive, RFP single positive, double labeled, and non-labeled. Importantly, the authors demonstrated that intra-myocardial injection of adenoviruses expressing $4 \mathrm{~F}$ following myocardial infarction increased single positive fluorescent clones to over $16 \%$ compared to $<1 \%$ in controls indicating progression through mitosis. In addition, 4F could also enhance cardiac function following myocardial injury. Myocardial infarction may promote productive $\mathrm{CM}$ proliferation, as the number of single positive mosaic clones was lower in uninjured hearts treated with $4 \mathrm{~F}$.

The exciting work by Mohammed et al. adds to the growing toolbox of gene products that enhance endogenous CM regeneration (Fig. 1). This is a growing list that includes cell cycle genes, genes encoding Hippo and Neuregulin signaling components, small non-coding RNAs, and extracellular matrix factors. ${ }^{4-8}$ The authors show that the precise combination of four cell cycle genes, or two factors combined with the appropriate small molecules, can promote CM proliferation. However, the use of non-cell type-specific adenoviruses by Mohammed et al., leading to $4 \mathrm{~F}$ expression in all cardiac cell types, raises many important questions. While the authors reported that $4 \mathrm{~F}$ overexpression had little effect on fibroblast proliferation, they did not address scar composition or scar resolution after injury. Further, potential effects of the $4 \mathrm{~F}$ cocktail on endothelial cells comprising the

\footnotetext{
${ }^{1}$ Program in Developmental Biology, Baylor College of Medicine, One Baylor Plaza, Houston, TX 77030, USA; ${ }^{2}$ Department of Molecular Physiology and Biophysics, Baylor College of Medicine, One Baylor Plaza, Houston, TX 77030, USA and ${ }^{3}$ Texas Heart Institute, Houston, TX 77030, USA

Correspondence: James F Martin (jfmartin@bcm.edu)
}

Received: 21 March 2018 Accepted: 28 March 2018

Published online: 19 April 2018 


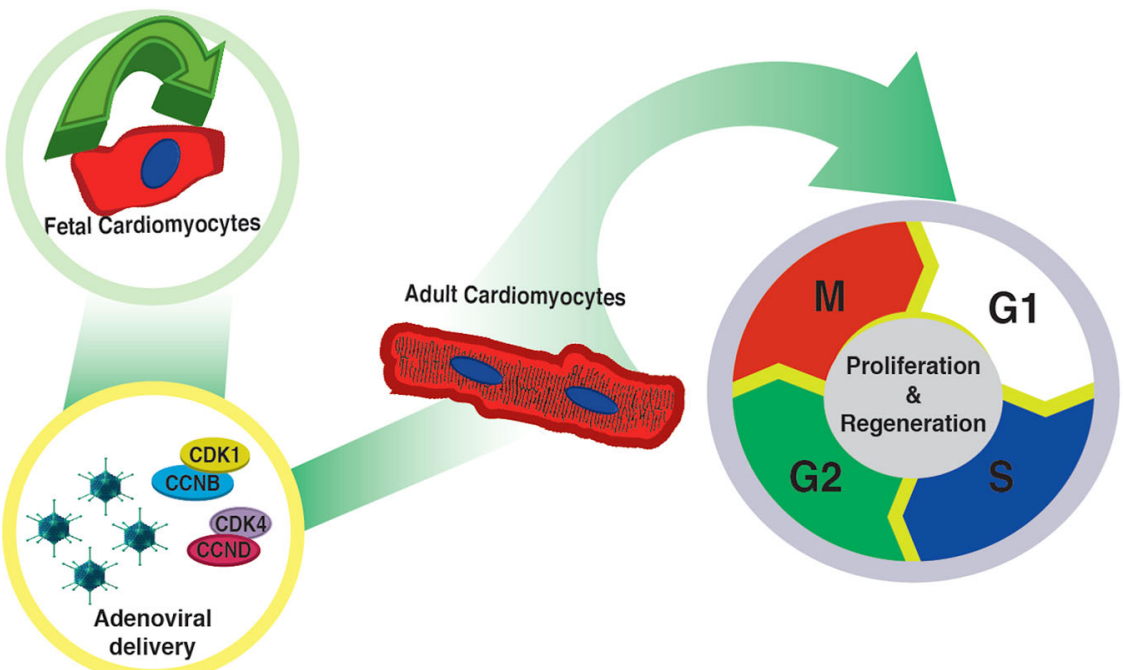

Fig. 1 Schematic diagram showing the strategy deployed by Mohamed et al. to boost adult CM proliferation. The authors determined the cell cycle regulators expressed in fetal and neonatal CMs, which possess proliferative capacity, and then through a series of screens determined which combinatorial cocktail of cell cycle regulators could induce mitosis in adult CMs in vivo following adenoviral delivery

vasculature and inflammatory myeloid cells need further investigation. Other important questions include: Is genome instability an issue for CMs? How does the sarcomere become re-organized during cell division? Overall, the $4 \mathrm{~F}$ cocktail defined by Mohammed et al. is a promising addition to potential therapies that may be useful for heart failure patients.

\section{REFERENCES}

1. Heallen, T. et al. Science 332, 458-461 (2011).

2. Quaife-Ryan, G. A. et al. Circulation 136, 1123-1139 (2017)

3. Espinosa, J. S., Tea, J. S. \& Luo, L. Cold Spring Harb. Protoc. 2014, 182-189 (2014).

4. Pasumarthi, K. B., Nakajima, H., Nakajima, H. O., Soonpaa, M. H. \& Field, L. J. Circ. Res. 96, 110-118 (2005).

5. Xin, M., Olson, E. N. \& Bassel-Duby, R. Nat. Rev. Mol. Cell. Biol. 14, 529-541 (2013).

6. Bassat, E. et al. Nature 547, 179-184 (2017).

7. Leach, J. P. et al. Nature 550, 260-264 (2017).

8. D'Uva, G. et al. Nat. Cell Biol. 17, 627-638 (2015). 